\title{
Law, Policy, and Syndromic Disease Surveillance: A Multi-Site Case Study
}

\author{
Jonathan Purtle*2, Robert Field ${ }^{1}$, Esther Chernak1, Tom Hipper ${ }^{1}$ and Jillian Nash ${ }^{1}$
}

${ }^{1}$ Drexel University Thomas R. Kline School of Law, Philadelphia, PA, USA, Philadelphia, PA, USA; ${ }^{2}$ Department of Health

Management \& Policy, Drexel University Dornsife School of Public Health, Philadelphia, PA, USA

\section{Objective}

Explore the impact of law and government policy on the practice of syndromic disease surveillance (SyS) in the United States.

\section{Introduction}

SyS systems have great potential to prevent morbidity, injury, and mortality by monitoring population health and providing realtime data to inform public health department decisions. Electronic health information technology and federal, state and local incentives and investments have helped to facilitate their rapid and widespread implementation. As a result, SyS systems operate in the context of laws and regulations that determine their success. An understanding of the effects of this legal environment is crucial to insuring that SyS systems fulfill their potential.

\section{Methods}

We conducted semi-structured, in-depth interviews with 55 SyS stakeholders (e.g., state/local health department officials, legal counsel, health care facility personnel) from six jurisdictions and facilitated focus groups with national SyS stakeholder organizations. All interviews and focus groups were audio-recorded, transcribed, and analyzed by two coders using thematic content analysis and NVivo software. We also identified and conducted a content analysis of relevant legal documents.

\section{Results}

Four main findings emerged. First, SyS was largely conducted under the same legal authority as 'traditional' (e.g., notifiable disease) public health surveillance. In some jurisdictions, statutes and administrative rules have been amended to include "disease clusters" and "syndromes" as notifiable conditions or to mandate reporting of emergency department data. In some jurisdictions, these regulations were promulgated at the request of health care facilities reporting SyS data that saw them as a legal safeguard.

Second, requirements for "Meaningful Use" of electronic health records promulgated under the Health Information Technology for Economic and Clinical Health Act have eased health care facilities' SyS-related legal concerns and injected funding that has substantially increased the number of facilities that send SyS data to state public health agencies and generally obviated the need for data use agreements. However, many public health agencies lack the staff to maximize the analytic potential of these data receive data only from emergency department and urgent care settings. Some public health professionals explained that Meaningful Use regulations lack a mechanism to maintain the quality of SyS data and that the incentive to satisfy Meaningful Use requirements has prompted some to outsource SyS reporting to third party vendors, weakening inter-personal relationships between health department and hospital personnel and hindering follow-up investigations as a result.

Third, very few legal concerns were expressed related to the federal BioSense program. Some jurisdictions participate in BioSense because they do not have the resources to conduct their own data collection and analysis, others do so to obtain financial resources for surveillance activities and to enhance the robustness of a national surveillance system. Officials in several jurisdictions that do not participate cited the indemnification clause of the BioSense data use agreement, which holds states liable in the event of a data breach, Others cited concerns regarding deidentification of the data.

Finally, we found that the primary barriers to maximizing the public health potential of SyS systems were technical issues and limited health department resources, not legal concerns. Although public health professionals, health care personnel, and public health legal professionals were aware of the legal context in which SyS practice operates, legal barriers were viewed as surmountable and secondary to limited information technology and epidemiology resources. In particular, few concerns were expressed regarding legal requirements for protecting data privacy, including those issued under the Health Insurance Portability and Accountability Act.

\section{Conclusions}

SyS has become an integral component of public health surveillance in the United States. Federal Meaningful Use electronic health record incentives and state public health statutes that mandate submission of data have facilitated the growth of these systems. They have also alleviated most of the legal concerns that had been raised when these systems were first established. The jurisdictions that participate in BioSense benefit from the financial and technical resources available through this program.

\section{Keywords}

Meaningful Use; BioSense; Law; Policy

\section{Acknowledgments}

Joe Gibson, Richard Hopkins, Jim Buehler, Laura Streichert, RWJF

\author{
*Jonathan Purtle \\ E-mail: jpp46@drexel.edu
}

